\title{
Manuelle Medizin aus Sicht der Anwender: Eine qualitative Studie mit Ärzten
}

\author{
Anne Knüpfer Stefanie Joos Katja Götz Jost Steinhäuser \\ Abteilung Allgemeinmedizin und Versorgungsforschung, Universitätsklinikum Heidelberg, Deutschland
}

\section{Schlüsselwörter}

Manuelle Medizin - Chirotherapie - Berufszufriedenheit . Qualitative Forschung $\cdot$ Komplementärmedizin

\section{Zusammenfassung}

Hintergrund: Manuelle Medizin (MM) hat einen hohen Stellenwert in der ambulanten Versorgung von Beschwerden des Bewegungsapparats. Trotz Vorliegen zahlreicher randomisiert-kontrollierter Studien und Meta-Analysen im Bereich der MM ist die Bewertung aufgrund verschiedener Schulen und Techniken teilweise schwierig. Ziel der vorliegenden Studie war es, bei der großen Beliebtheit dieser Therapieform die Motivation und Erfahrungen von Ärzten bezüglich der Anwendung von MM in der täglichen Praxis zu ergründen. Methoden: Im Rahmen eines qualitativen Forschungsansatzes wurden 21 semi-strukturierte, telefonische Einzelinterviews mit Ärzten mit der Zusatzqualifikation für MM durchgeführt. Die Rekrutierung erfolgte über die Deutsche Gesellschaft für Manuelle Medizin sowie über einen Verteiler von Ärzten, die an Forschungsfragen zu MM interessiert sind. Die Interviews wurden aufgezeichnet, transkribiert und, unterstützt durch das Computerprogramm Atlas.ti, inhaltsanalytisch ausgewertet. Ergebnisse: Als Hauptmotivation für die Anwendung von MM wurde eine Stärkung der Arzt-Patienten-Beziehung durch einen unmittelbaren Zugang zum Patienten mit schnellen Behandlungserfolgen geschildert. Weitere beschriebene Aspekte sind die Einfachheit der Methode, die Unabhängigkeit von räumlichen oder apparativen Gegebenheiten und die damit verbundenen niedrigen Kosten. Positive, als direkt die Zufriedenheit mit MM beeinflussend beschriebene Faktoren sind die Möglichkeit, eigene Fähigkeiten zu nutzen, den Patienten zu einem gesunden Lebensstil zu motivieren und die Dankbarkeit des Patienten. Als Aspekte, welche die Zufriedenheit negativ beeinflussen, wurden die schlechte Vergütung der Therapie, die unklare Evidenzlage, die Anwendung bei oft selbstlimitierenden Krankheiten und das Risiko schwerwiegender Nebenwirkungen sowie die Gefahr einer iatrogenen Fixierung genannt. Schlussfolgerungen: Die Ergebnisse dieser Studie zeigen eine große Bandbreite von zumeist positiven Sichtweisen und Erfahrungen von Ärzten bei der Anwendung von MM. Dem Wunsch nach dem unmittelbar heilenden Handeln scheint durch MM begegnet zu werden. Die MM scheint als willkommene Abwechslung zur alltäglichen Berufsausübung zu dienen, die als distanziert wahrgenommen wird.

\author{
Keywords \\ Manual medicine $\cdot$ Chiropractic $\cdot$ Job satisfaction . \\ Qualitative research . Complementary medicine
}

\section{Summary \\ Manual Medicine from the User's Perspective: A Qualitative Study with Physicians}

Background: Manual medicine (MM) has high importance in the ambulant treatment of complaints of the musculoskeletal system. Although there are several randomized controlled trials and meta-analyses, evidence about its efficacy is limited due to different organizations offering MM courses teaching different techniques. The aim of this study was to gain an understanding of the motivation and experiences of physicians using MM in daily practice. Methods: In a qualitative study, 21 semi-structured phone call interviews were performed with physicians who have an additional qualification in MM. Recruitment was done by sending an e-mail to every physician listed on the homepage of the German Society for Manual Medicine or known to be interested in $\mathrm{MM}$ research from a previous study. Interviews were performed, recorded, transcribed and evaluated content-analytically using the software program Atlas.ti. Results: One of the main motivations for using MM therapy was a better doctor-patient relationship because of improved patient access, with often rapid treatment success. Further advantages were the relative simplicity of the method, the independence from spatial or apparatus conditions and the associated low costs. Positive factors discussed as directly influencing the satisfaction with MM are the possibility to use own skills, the gratitude of the patient, and the possibility to influence the patient towards a healthy lifestyle. Factors negatively influencing the satisfaction were named as follows: the low gratification for the therapy, heterogeneous evidence, the indication in often self-limiting diseases, the risk of serious adverse effects and the risk of iatrogenic fixation. Conclusions: The results of this study show a wide range of mostly positive views and experiences of physicians when applying MM. The immediate curative action seems to be a welcome change for physicians who perceive the practice of their profession seen as distant from the patient.

\section{KARGER}

Fax +497614520714

Information@Karger.de

www.karger.com (c) 2012 S. Karger GmbH, Freiburg

1661-4119/12/0193-0137\$38.00/0

Accessible online at:

www.karger.com/fok
Dr. med. Jost Steinhäuser

Abteilung Allgemeinmedizin und Versorgungsforschun

Universitätsklinikum Heidelberg

Voßstraße 2, 69115 Heidelberg, Deutschland

Tel. +49 6221 56-8295, Fax -1972

jost.steinhaeuser@med.uni-heidelberg.de 


\section{Einleitung}

Die Manuelle Medizin (MM) hat in der ambulanten Versorgung von Beschwerden des Bewegungsapparats national und international in den letzten Jahren zunehmend an Bedeutung gewonnen. Die Anzahl der Ärzte mit der Zusatzbezeichnung MM hat sich in den letzten 15 Jahren annähernd verdreifacht (von 7175 im Jahr 1995 auf 19409 im Jahr 2010) [1]. In einer bundesweiten Umfragestudie bei Hausärzten gehörte die MM zu den 4 am häufigsten eingesetzten komplementärmedizinischen Therapieformen in der täglichen Praxis [2]. Darüber hinaus wurde sowohl in der genannten Studie als auch im Rahmen einer Internetbefragung bei Ärzten, die MM anwenden, ein hoher Grad an Zufriedenheit mit der Methode erhoben $[2,3]$. Die Therapie mit MM ist als Leistung der gesetzlichen Krankenversicherung abrechenbar [4]. Schwerpunkte für das Anwenden von MM sind Schmerzen des Bewegungsapparats, insbesondere des Iliosakralgelenks, der Halswirbelsäule, der Lendenwirbelsäule und der Brustwirbelsäule [3].

So unterschiedlich die Anwendungsgebiete sind, so vielfältig sind auch die therapeutischen Herangehensweisen. Sowohl in Deutschland als auch international gibt es ein vielfältiges Angebot an Schulen bzw. Institutionen, die in ihren Kursen jeweils unterschiedliche diagnostische und therapeutische Techniken vermitteln [5]. So unterscheidet sich je nach Schule die Diagnostik dahingehend, dass beispielsweise eine Meridiandiagnostik mit einbezogen wird oder nicht. In der Therapie gibt es grundlegende Unterschiede bereits in der Lagerung von Patienten z.B. mit einer Blockade eines Costotransversal-Gelenks [6, 7]. Allen Techniken gemeinsam ist, dass sie unmittelbar durch den Therapeuten, zumeist mit den Händen und ohne weitere Hilfsmittel ausgeführt werden.

Die heterogene Praxisrealität spiegelt sich auch in der schwer fassbaren Evidenzlage wider. Nach dem von Bronfort et al. publizierten UK-Evidenzbericht [8] ist MM bei akuten und chronischen Kreuzschmerzen, Nackenschmerzen, Migräne, zervikogenem Kopfschmerz und Schwindel sowie bei Beschwerden der Extremitätengelenke wirksam.

Im Hinblick auf die Behandlung von Kreuzschmerzen, einem der Hauptanwendungsbereiche der MM, kommen verschiedene Reviews zu dem Schluss, dass MM anderen gängigen Therapien ebenbürtig ist $[9,10]$. Neben der medizinischen Wirksamkeit wurde auch die Kosteneffektivität von MM bei Kreuzschmerzen beschrieben [11]. In der deutschen Nationalen Versorgungsleitlinie wurde der MM bei Kreuzschmerzen eine «Kann-Empfehlung» zugesprochen [12]. Dieser Empfehlungsgrad erscheint, verglichen mit der in der Literatur beschriebenen Zufriedenheit beim Anwenden von MM und der weiten Verbreitung der MM, als eher gering [2, 3, 12]. Ziel der vorliegenden Studie war es daher, die Motivation und Erfahrungen von Ärzten bezüglich der Anwendung von MM in der täglichen Praxis zu ergründen.

\section{Methoden}

Für die vorliegende Studie wurde ein qualitatives Design gewählt, um ein grundlegendes Verständnis über individuelle Motive und Verhalten sowie Einblicke in die subjektiven Einstellungen und Erfahrungen der Ärzte zu erlangen. Qualitative Forschung erhebt nicht den Anspruch auf Repräsentativität der Daten, allerdings auf intersubjektive Nachvollziehbarkeit mit dem Ziel, Hypothesen zu generieren [13]. Interviewt wurden ausschließlich Ärzte, die die Zusatzbezeichnung für MM besitzen. Als Methode zur Datenerhebung wurden halbstrukturierte, leitfadengeführte Interviews gewählt, deren Vorteil darin liegt, strukturierte Informationen über die subjektive Sichtweise der Interviewten zu erhalten. Im Rahmen halbstrukturierter Interviews kann direkt auf die einzelnen Antworten der Ärzte eingegangen und gegebenenfalls in die Tiefe gefragt werden. Im Leitfaden wurden offene und geschlossene Fragen eingebracht, um sowohl konkrete Antworten zu erlangen als auch subjektive Einstellungen und Erfahrungen erfassen zu können. Die Leitfragen wurden durch Kenntnisse vorangehender Studien im Bereich der MM bzw. Komplementärmedizin [2,3] sowie aus der praktischen Tätigkeit der Autoren entwickelt.

Die Themenbereiche der Interviewleitfragen umfassen Motivation, Stellenwert der MM, Indikationen und Patienten sowie Procedere in der Praxis.

Jeder Themenbereich beinhaltet verschiedene Leitfragen. Da in der vorliegenden Studie der Fokus auf der Motivation bezüglich der Anwendung von MM liegt, werden nur die Fragen aus diesem Bereich dargestellt: «Wenden Sie MM gerne an?», «Wenn ja, warum?» und «Was macht Sie beim Anwenden von MM zufrieden?»

\section{Rekrutierung der Ärzte}

Zur Teilnahme an der Studie wurden in Deutschland tätige Ärzte mit der Zusatzbezeichnung für MM eingeladen. Die Einladung bzw. Rekrutierung der Interviewprobanden erfolgte auf 2 Wegen. 141 Ärzte der Fechterschule, die durch eine frühere Studie als an manualmedizinischen Forschungsfragen interessiert bekannt waren, wurden per E-Mail angeschrieben; ebenso wie die 80 Ärzte, die auf der Website der Deutschen Gesellschaft für MM (DGMM) mit ihrer E-Mail-Adresse verzeichnet waren [14]. Bei Interesse an der Studie wurden weitere Informationen zur Verfügung gestellt und eine Teilnahmeerklärung versandt.

\section{Durchführung}

Nach Vorliegen der Teilnahmeerklärung wurde ein Interviewtermin vereinbart. Die Interviews wurden mittels Computer aufgezeichnet. Die Dauer der Telefoninterviews lag zwischen 9 und 38 min (Mittelwert 19 min). Anschließend wurden die Telefoninterviews pseudonymisiert, transkribiert und inhaltsanalytisch ausgewertet.

\section{Datenaufbereitung und Auswertung}

Die Auswertung erfolgte mithilfe der qualitativen Inhaltsanalyse nach Philipp Mayring und wurde durch das Softwareprogramm Atlas.ti 5.2.17 (Scientific Software Development GmbH, Berlin, Deutschland) unterstützt [15]. Um die intersubjektive Nachvollziehbarkeit gemäß den Gütekriterien qualitativer Forschung zu bewahren [13], wurden unabhängig voneinander durch mehrere an der Auswertung beteiligte Personen Kategorien entwickelt. Anschließend wurden im Sinne einer konsensuellen Kodierung die Zuordnungen verglichen und diskutiert. Die Kategorienbildung fand zunächst deduktiv auf Basis der Leitfragen statt. Im weiteren Verlauf wurde das Kategoriensystem induktiv erweitert. Nach 21 Interviews war eine inhaltliche Sättigung zu erkennen, sodass keine weiteren Rekrutierungsbemühungen mehr durchgeführt wurden. Es wurden prägnante Aussagen der Ärzte aus der jeweiligen Kategorie zur Veranschaulichung ausgewählt und generalisiert. 


\section{Ergebnisse}

21 Ärzte aus unterschiedlichen Regionen Deutschlands nahmen an den Interviews teil. Eine Übersicht der Teilnehmer ist in Tabelle 1 zusammengestellt.

\section{Anwendung von MM}

Die Mehrzahl der interviewten Ärzte wendet MM gern an. Die mit den Interviews erfassten Aspekte zur Motivation mit Bezug auf die Anwendung von MM lassen sich in folgende Hauptkategorien einteilen: die Arzt-Patienten-Beziehung, die Möglichkeit zur Diagnostik sowie den Therapieerfolg (Tab. 2).

\section{Arzt-Patienten-Beziehung}

Ein wesentlicher Grund für die Anwendung von MM in der Patientenversorgung, den die Teilnehmer berichten, stellt der direkte Körperkontakt zu den Patienten dar, der dem Arzt zu einem besseren Zugang zum Patienten verhilft. Die körperliche Nähe erwecke Vertrauen und verbessere, so die Meinung der Ärzte, das Arzt-Patienten-Verhältnis.

«Das ist auch ein Kontakt zum Patienten, der allein durch auch die Haptik, durch das Anfassen natürlich nicht nur 'ne reine technische Untersuchung ist, sondern auch Patientenvertrauen durchaus fördert» (A20).

Ein weiterer häufig genannter Aspekt im Rahmen der Anwendung ist der erleichterte Zugang zur Psyche des Patienten durch den direkten Körperkontakt. Im Rahmen dieses unmittelbaren Kontakts können «Verspannungen», deren Ursache im psychischen Bereich vermutet wird, besser thematisiert werden. Ergänzend wird die Möglichkeit, unmittelbar etwas bewirken zu können, als bereichernd empfunden, im Gegensatz beispielsweise zur Ausstellung eines Rezepts, die mehrfach als beliebig und distanziert geschildert wurde.

«Man kann auch spüren, wie ängstlich ein Mensch ist oder was der so an Abwehr in seinem Körper hat, wo der wirklich verspannt ist. Und wenn man mit einem Bleistift und 'nem Rezept dem Menschen gegenüber sitzt mit der Distanz des Schreibtischs dazwischen, dann kann man einen Menschen nicht wahrnehmen und nicht spüren. Und das geht in der heutigen Medizin ganz häufig verloren» (A18).

\section{Möglichkeit zur Diagnostik}

In den Telefoninterviews wurden mehrmals als Begründung für die Anwendung von MM die Einfachheit der Methode und die Unabhängigkeit von anderen Fachkollegen sowie von apparativer Diagnostik betont.

«Dass ich 'ne direkte Diagnostikmethode und Behandlungsmethode habe, ohne dass ich noch jemanden konsultieren muss oder 'nen Rezept schreiben oder sonst was» (A13).

Ebenfalls sei die MM eine der wenigen medizinischen Diagnostik- und Therapiemethoden, die überall anwendbar sei.
Tab. 1. Soziodemographische Zusammensetzung der Studienteilnehmer $(\mathrm{N}=21)$

\begin{tabular}{lc}
\hline Soziodemographische Variablen & $\mathrm{n}(\%)$ \\
\hline Geschlecht & $3(14)$ \\
Weiblich & $18(86)$ \\
Männlich & $52(34 / 78$ Jahre) \\
Alter, MW (Min/Max) & \\
Facharztbezeichnung & $10(48)$ \\
Allgemeinmedizin & $7(33)$ \\
Orthopädie & $4(19)$ \\
Sonstiges (HNO, PRM, NM usw.) & \\
Schule & $10(48)$ \\
MWE & $6(28,5)$ \\
Fechter & $3(14)$ \\
ÄMM & $2(9,5)$ \\
FAC & \\
Tätigkeitsort & \\
Einzelpraxis & $5(24)$ \\
Gemeinschaftspraxis & $8(38)$ \\
Krankenhaus & $4(19)$ \\
Sonstiges & $4(19)$ \\
Ort & \\
Land & \\
\hline
\end{tabular}

MW = Mittelwert; HNO = Hals-Nasen-Ohren-Heilkunde; PRM = Physikalische Medizin und Rehabilitation; NM = Nuklearmedizin; MWE = Dr. Karl Sell Ärzteseminar Neutrauchburg e.V.; ÄMM = Ärztevereinigung für Manuelle Medizin - Ärzteseminar Berlin e.V.; FAC $=$ Ärzteseminar Hamm - Boppard e.V.

Tab. 2. Das Kategoriensystem zum Themenbereich «Motivation»

\begin{tabular}{|c|c|}
\hline Hauptkategorie & Unterkategorie \\
\hline \multicolumn{2}{|l|}{ Anwendung von MM } \\
\hline \multirow{3}{*}{ Arzt-Patienten-Beziehung } & direkter Körperkontakt \\
\hline & Zugang zum Patienten \\
\hline & Förderung von Vertrauen \\
\hline \multirow[t]{2}{*}{ Möglichkeit zur Diagnostik } & Unabhängigkeit \\
\hline & Einfachheit \\
\hline \multirow[t]{5}{*}{ Therapieerfolg } & geringer Zeitaufwand \\
\hline & geringe Kosten \\
\hline & schneller Wirkungseintritt \\
\hline & hohe Effektivität \\
\hline & geringe Nebenwirkungen \\
\hline \multicolumn{2}{|l|}{ Zufriedenheit mit MM } \\
\hline \multirow[t]{4}{*}{ Positiv beeinflussend } & Spaß an der Methode \\
\hline & eigene Aktivität \\
\hline & Dankbarkeit des Patienten \\
\hline & Motivierung des Patienten \\
\hline \multirow[t]{5}{*}{ Negativ beeinflussend } & selbstlimitierende Erkrankungen \\
\hline & schwerwiegende Nebenwirkungen \\
\hline & geringe Vergütung \\
\hline & fehlende Evidenz \\
\hline & iatrogene Fixierung \\
\hline
\end{tabular}


«Ich bin unabhängig. (...) Ich kann sie wirklich überall anwenden. Einer meiner Lehrer hat mal gesagt: auf'm Nordpol und in der Sahara. Und das sind nur sehr wenige medizinische Verfahren, die ohne Räume, ohne Geräte, nur mit den Händen allein Diagnose machen und Therapie machen» (A3).

\section{Therapieerfolg}

In den Interviews wurde besonders hervorgehoben, dass die Anwendung von MM zu einem schnell eintretenden Therapieerfolg führen kann.

«Der erzielte Effekt. Also man sieht es sofort, dass die Patienten sich wieder besser bewegen können. Dass sie einfach wieder beschwerdefrei oder mindestens beschwerdegelindert sind» (A9).

Die Effektivität der MM wird so hoch eingeschätzt, dass man durch MM Schmerzmedikamente einsparen und Patienten sogar gelegentlich eine Operation ersparen könne. Mehrmals wurde zudem angegeben, dass die Nebenwirkungen der MM wesentlich geringer seien im Vergleich zu einer Behandlung mit nichtsteroidalen Antirheumatika (NSAR).

«Ohne dem Patienten dabei Schaden zuzufügen. Ohne aufschneiden zu müssen und nachzugucken. Das brauch ich nicht. Das ist mir sehr angenehm, dass ich keine Narben hinterlasse» (A17).

Des Weiteren wurden die durch das Anwenden von MM entstehenden geringen Kosten genannt.

«Ja, das wäre natürlich volkswirtschaftlich, politisch das Ideale, weil die Manualtherapie kostet ja ganz, ganz wenig im Vergleich zu dem, was die Orthopäden sonst an Kosten verursachen. (...) Ich hab also keine Regressprobleme mit Arzneimittelbudget und so weiter» (A8).

\section{Zufriedenheit mit MM}

Die Zufriedenheit bei der Anwendung von MM wird nach Angaben der Teilnehmer sowohl positiv als auch negativ beeinflusst.

\section{Positive Einflüsse auf die Zufriedenheit}

Die Mehrheit der interviewten Ärzte ist mit der Anwendung von MM zufrieden. Faktoren wie Spaß an der Methode, Stolz auf das eigene Können und geschickte Hände wurden hierbei erwähnt. Als ergänzender Aspekt scheint die bei der Therapie notwendige eigene Aktivität die Zufriedenheit $\mathrm{zu}$ erhöhen.

«Außerdem ist man selbst gut in Bewegung. Das ist auch noch so ein Aspekt, ja, der für mich wichtig war» (A9).

Die Dankbarkeit von erfolgreich behandelten Patienten fördere ebenfalls die Zufriedenheit der Ärzte und wird somit als besondere Motivation erfahren.

«Und wenn ich's jetzt mal nur auf den Patienten beziehe, die Zufriedenheit, die Dankbarkeit, dass ich also einen akuten Schmerz, so er denn eben durch eine funktionelle Störung ausgelöst ist, sehr rasch und sehr schmerzarm und sehr befriedigend beseitigen $\mathrm{zu}$ können, einen glücklichen Patienten nach Hause entlassen zu können» (A2).

Durch den verbesserten Zugang zum Patienten hoffen einige Ärzte, die Patienten auch in ihrer Lebensführung positiv beeinflussen zu können. So steige die Chance, den Patienten beispielsweise über körperliches Training zur Selbstwirksamkeit aktivieren und damit zur eigenen Vorsorge vor weiteren Beschwerden motivieren zu können.

«Und es bezieht den Patienten dann im zweiten Schritt eben auch aktiv mit ein. Das find ich zum Beispiel total wichtig. Also es gibt zumindest die Möglichkeit. Viele Leute wachen dann auch auf und merken, ah ja, so'n bisschen Training, das hilft ja auch ganz gut, und bleiben dann vielleicht dran und haben dann auch dauerhaft weniger Probleme. Das wäre ja das Ziel, um da irgendwo 'nen Samen zu setzen. Und nicht immer nur dann, wenn's gar nicht mehr geht, zum Arzt zu kommen und sich irgend 'ne Behandlung abzuholen» (A1).

\section{Negative Einflüsse auf die Zufriedenheit}

Kritisch wurde von einigen Ärzten angemerkt, dass MM häufig bei banalen, selbstlimitierenden Erkrankungen eingesetzt wird.

«Die Krankheitsbilder, die mit Manueller Medizin behandelt werden oder mit Chirotherapie, sind ja meistens banale Erkrankungen, die selbstlimitierend sind» (A21).

In diesem Zusammenhang wurde auch das Problem der iatrogenen Fixierung, der Fixierung des Patienten auf den Arzt, von einigen Ärzten kritisch thematisiert. Einerseits erlebe sich der Arzt durch seine individuellen therapeutischen Fähigkeiten als einzigartig, andererseits beinhalte diese Fixierung insbesondere bei Patienten mit Bagatellerkrankungen einen deutlich negativen Aspekt.

«Was ich beobachtet habe, ist, dass man Knacksüchtige züchten kann. Also chirotherapieabhängige Patienten, meistens hypermobile junge Frauen» (A18).

Als weiterer negativ beeinflussender Faktor im Kontext der MM wurde die Gefahr schwerwiegender Nebenwirkungen, wie z.B. der Vertebralisdissektion, genannt. Als Konsequenz berichteten einzelne Ärzte, dass sie insbesondere bei älteren Patienten mit gesundheitlicher Vorbelastung von MM Abstand nehmen.

«Und dann natürlich die schwerwiegenden Argumente an der Halswirbelsäule (...) die Gefahr der Vertebralisdissektion» (A12).

Weiterhin wurden die als zu gering erlebte Vergütung der MM-Therapie sowie die für die verschiedenen Indikationen unterschiedlich ausgeprägte wissenschaftliche Evidenz negativ erwähnt.

\section{Diskussion}

In den Interviews wurden neben den Gründen der Anwendung von MM auch die positiven und negativen Einflüsse auf 
die Zufriedenheit mit MM beschrieben. Hauptgründe für die Anwendung von MM waren der durch den direkten Körperkontakt verbesserte Zugang zum Patienten, die Einfachheit und Unabhängigkeit der Methode und der erlebte Therapieerfolg. Allein die Anwendung von MM in der Praxis scheint die Zufriedenheit und Arbeitsmotivation der behandelnden Ärzte zu erhöhen. Diese wird allerdings negativ beeinflusst durch die schlechte Vergütung der Therapie, die oftmals banalen Krankheitsbilder, für die die Therapie eingefordert wird, das Risiko schwerwiegender Nebenwirkungen, die Gefahr der iatrogenen Fixierung und die zum Teil fehlende Evidenz.

In den Interviews wurden von einigen Teilnehmern die Risiken von MM denen von NSAR gegenübergestellt; dabei wurde angegeben, dass die erlebten Nebenwirkungen der $\mathrm{MM}$ wesentlich geringer seien als diejenigen von NSAR. Zu diesem Schluss, dass das Anwenden von MM wesentlich sicherer ist als die Anwendung von NSAR, kamen auch Dabbs und Lauretti in einer Studie mit Patienten mit Nackenschmerzen [16]. Einer in Deutschland durchgeführten Umfragestudie zufolge verordnen allerdings 69\% der Ärzte zusätzlich zur MM-Behandlung NSAR [3]. Dies ist kritisch zu bewerten, da durch das gleichzeitige Anwenden von MM und Medikamenten vermehrt Komplikationen auftreten können [17]. Zukünftige Studien sollten erheben, warum trotz des Einsatzes von MM in einem so hohen Ausmaß NSAR ergänzend eingesetzt werden. Ein oft in den Interviews erwähnter Aspekt ist, dass Diagnostik und Therapie im Rahmen der MM ohne zusätzliche Geräte und Konsiliaruntersuchungen auskommen und die MM damit eine kostengünstige Therapiemethode darstellt, was sich auch in der aktuellen Studienlage zur Kosteneffektivität von MM widerspiegelt [11]. Im Kontrast zu dieser Kosteneffektivität steht die als schlecht erlebte Vergütung der MM. Hier müssten zukünftig ökonomische Evaluationen die MM mit anderen Therapieverfahren vergleichen.

Einige der interviewten Ärzte bemerken durch die MM eine iatrogene Fixierung des Patienten und erleben dies als negativ, da sich die Beschwerden des Patienten durch die Abhängigkeit vom Arzt oftmals chronifizieren und somit keine «Heilung» erreicht werden kann.

Allerdings empfinden andere Interviewteilnehmer gerade das unmittelbare und individuelle Behandeln als eine Bereicherung des Praxisalltags, was mit einer erlebt hohen Dankbarkeit des Patienten einhergeht. Hier scheinen die Grenzen zwischen dem Aufwerten des Arztes und der Fixierung des Patienten fließend zu verlaufen.

Die hohe Zufriedenheit mit der Anwendung von MM scheint positive Auswirkungen sowohl auf die Arbeitsmotivation als auch auf die Berufszufriedenheit zu haben. Wenn diese Hypothese in zukünftigen Studien bestätigt werden kann, könnte die MM einen wichtigen Einfluss auf die Qualität der Patientenversorgung haben [18]. Diesbezüglich konnte bereits 2011 ein Zusammenhang zwischen einer hohen Arbeitszufriedenheit und dem Anwenden von Komplementärmedizin bei einer positiven Einstellung gegenüber der Komplementärmedizin beschrieben werden [19]. Die Möglichkeit, die eigenen Fähigkeiten zu nutzen, wurde neben der Dankbarkeit und der Aktivierungsmöglichkeit des Patienten in den Interviews als ein Faktor genannt, der die Zufriedenheit beim Anwenden der MM positiv beeinflusst. Diese Aussage passt zu den Ergebnissen einer Studie, ebenfalls aus dem Jahr 2011, in der die Möglichkeit, die eigenen Fähigkeiten anzuwenden, als wichtigster Faktor für die Arbeitszufriedenheit von Allgemeinmedizinern identifiziert wurde [20].

\section{Stärken und Schwächen}

Diese Studie widmet sich erstmalig möglichen Hintergründen für die weit verbreitete Anwendung von MM aus Therapeutensicht.

Für die Studie wurden bewusst Ärzte mit Erfahrung im Bereich MM rekrutiert. Erwartungsgemäß äußerten sich diese Ärzte eher positiv über die MM, obwohl auch einige negative Aspekte genannt wurden. Selbstverständlich kann durch dieses Selektionsbias sowie durch die niedrige Anzahl eingeschlossener Ärzte kein Rückschluss auf die Gesamtpopulation der MM anwendenden Ärzte gezogen werden. Repräsentativität ist allerdings nicht das Ziel der verwendeten qualitativen Methode, bei der methodenbedingt keine statistischen Aussagen getroffen werden können, sondern Hypothesen generiert werden sollen.

\section{Fazit für die Praxis}

Die Ergebnisse dieser Studie zeigen eine große Vielfalt von zumeist positiven Aspekten und Erfahrungen von Ärzten bei der Anwendung der MM. Die MM scheint den Ärzten durch die unmittelbare Kontaktaufnahme eine bessere Arzt-PatientenBeziehung zu ermöglichen. Der Wunsch nach dem unmittelbar heilenden Handeln scheint durch die MM erfüllt zu werden. Die MM dient zudem als willkommene Abwechslung zur alltäglichen Berufsausübung, die als distanziert wahrgenommen wird.

\section{Disclosure Statement}

Bei keinem der Autoren besteht ein Interessenkonflikt. 


\section{Literatur}

1 Gesundheitsberichterstattung des Bundes: Bei den Ärztekammern registrierte Ärztinnen und Ärzte mit Zusatz-Weiterbildungen (Zusatzbezeichnung). Gliederungsmerkmale: Jahre, Region, Geschlecht, Zusatz-Weiterbildung, Tätigkeitsbereich. $w w w$. gbe-bund.de/oowa921-install/servlet/oowa/aw92/ dboowasys921.xwdevkit/xwd_init?gbe.isgbetol/xs_ start_neu/\&p_aid=i\&p_aid=36589900\&nummer $=612 \& p \_s p r a c h e=D \& p \_i n d s p=-\& p \_a i d=52957048$ (Zugriff am 23.05.2012)

2 Joos S, Musselmann B, Szecsenyi J: Integration of complementary and alternative medicine into family practices in Germany: results of a national survey. Evid Based Complement Alternat Med 2009; doi 10.1093/ecam/nep019.

3 Steinhäuser J, Oser A, Götz K, Joos S: Manuelle Medizin in Deutschland - eine deskriptive Analyse. Orthopäde 2011;40:339-343.

4 www.kbv.de/ebm2012/ebmgesamt.htm.

5 Smolenski UC: Wissenschaftskultur in der Manuellen Medizin. Manuelle Med 2002;40:323-324.

6 Fechter L: Chirotherapie in Bildern. Leer, Sollermann, 2005.

7 Bischoff HP, Moll H: Kurz gefasstes Lehrbuch der Manuellen Medizin. Balingen, Spitta, 2007.
Bronfort G, Haas M, Evans R, Leininger B, Triano J: Effectiveness of manual therapies: the UK evidence report. Chiropr Osteopat 2010;18:3.

$\checkmark$ Arthus M, Van der Windt DA, Jordan KP, Hay EM: Low back pain symptoms show a similar pattern of improvement following a wide range of primary care treatments: a systematic review of randomized clinical trials. Rheumatology 2010;49: 2346-2356.

10 Ernst E, Canter PH: A systematic review of systematic reviews of spinal manipulation. J R Soc Med 2006;99:192-196.

11 Lin CWC, Haas M, Maher CG, Machado LAC, Tulder MW: Cost-effectiveness of guidelineendorsed treatments for low back pain: a systematic review. Eur Spine J 2011;20:1024-1038.

12 Nationale Versorgungsleitlinie Kreuzschmerzen. $w w w$.versorgungsleitlinien.de/themen/kreuzschmerz/pdf/nvl_kreuzschmerz_lang.pdf.

3 Barbour RS: Checklist for improving rigour in qualitative research: a case of the tail wagging the dog? BMJ 2001;322:1115-1117.

14 Deutsche Gesellschaft für Manuelle Medizin. www. dgmm.de/index.php?option $=$ com_content und view $=$ article und $i d=127$ :mitgliederverzeichnis und catid $=48$ :home und Itemid $=98$.
15 Mayring P: Qualitative Inhaltsanalyse. Grundlagen und Techniken, ed 11. Weinheim, Beltz, 2010.

16 Dabbs V, Lauretti WJ: A risk assessment of cervical manipulation vs. NSAIDs for the treatment of neck pain. J Manipulative Physiol Ther 1995;18: 530-536.

17 Carnes D, Mars TS, Mullinger B, Froud R, Underwood M: Adverse events and manual therapy: A systematic review. Man Ther 2010;15:355-363.

18 Linzer M, Baier Manwell L, Williams ES, et al. Working conditions in primary care: physician reactions and care quality. Ann Intern Med 2009;151: 28-36.

19 Joos S, Musselmann B, Szecsenyi J, Goetz K: Characteristics and job satisfaction of general practitioners using complementary and alternative medicine in Germany - is there a pattern? BMC Complement Altern Med 2011;11:131.

20 Goetz K, Campbell SM, Steinhäuser J, et al.: Evaluation of job satisfaction of practice staff and general practitioners: an exploratory study. BMC Fam Pract 2011;12:137. 